\title{
The rhetoric and reality of integrated patient-centered care for healthcare providers: An ethnographic exploration of epilepsy care in Ireland
}

\author{
John-Paul Byrne ${ }^{\text {a }}$, Robert Power ${ }^{\text {b }}$, Rachel Kiersey ${ }^{\text {b }}$, Jarlath Varley ${ }^{\text {b }}$, Colin P. Doherty ${ }^{\text {c,d }}$, A. Jamie Saris ${ }^{\text {a }}$, \\ Veronica Lambert ${ }^{\mathrm{e}}$, Mary Fitzsimons ${ }^{\mathrm{b}, *}$ \\ a Department of Anthropology, National University of Ireland (NUI) Maynooth, Maynooth, Co. Kildare, Ireland \\ ${ }^{\mathrm{b}}$ Research and Innovation, The Royal College of Surgeons in Ireland, 111 St. Stephen's Green, Ardilaun House, Block B Second Floor, St Stephen's Green, Dublin 2, Ireland \\ ' Department of Neurology, St. James's Hospital, James's Street, Dublin 8, Ireland \\ d School of Medicine, Trinity College, Dublin 2, Ireland \\ e School of Nursing and Human Sciences, Dublin City University, Dublin 9, Ireland
}

\section{A R T I C L E I N F O}

\section{Article history:}

Received 6 February 2019

Accepted 8 February 2019

Available online 18 March 2019

\section{Keywords:}

Ethnographic research

Beliefs

Person-centered care

Health professionals in epilepsy care

Integrated care

Chronic disease

\begin{abstract}
A B S T R A C T
In line with healthcare reform across the world, the National Clinical Programme for Epilepsy (NCPE) in Ireland describes a model that aims to achieve holistic integrated person (patient)-centered care (PCC). While generally welcomed by stakeholders, the steps required to realize the NCPE ambition and the preparedness of those involved to make the journey are not clear. This study explored the perceptions of healthcare providers in the Irish epilepsy care ecosystem to understand their level of readiness to realize the benefits of an integrated PCC model. Ethnographic fieldwork including observations of different clinical settings across three regions in Ireland and one-to-one interviews with consultant epileptologists $(n=3)$, epilepsy specialist nurses $(n=5)$, general practitioners $(n=4)$, and senior healthcare managers $(n=3)$ were conducted. While there is a person-centered ambiance and a disposition toward advancing integrated PCC, there are limits to the readiness of the epilepsy care environment to fully meet the aspirations of healthcare reform. These are the following: underdeveloped healthcare partnerships;, poor care coordination; , unintended consequences of innovation;, and tension between pace and productivity. In the journey from policy to practice, the following multiple tensions collide: policy aims to improve services for all patients while simultaneously individualizing care; demands for productivity limit the time and space required to engage in incremental and iterative improvement initiatives. Understanding these tensions is an essential first step on the pathway to integrated PCC implementation.
\end{abstract}

(C) 2019 Elsevier Inc. All rights reserved.

\section{Introduction}

Modernization of healthcare systems across the world is aiming to move from simply treating illness to also promoting and maintaining health and well-being. Achieving this balance requires a reform from traditional episodic, reactive, hospital-centric healthcare to a modern integrated model of person (patient)-centered care (PCC) [1-10]. Integrated PCC is about ensuring that individuals have timely access to safe and efficient quality health services that meet their life-course needs and are coordinated across a continuum of care [11]. It also involves a shift from medical paternalism to a more interpersonal collaborative relationship between patient and (health) carer [12-14]. The overall ambition of integrated PCC is the realization of the quadruple aim of enhancing patient experience, improving population health, reducing costs, and improving the working life of healthcare providers (HCPs) $[6,7,9,15]$.

* Corresponding author at: RCSI Institute of Research, Royal College of Surgeons in Ireland, Ardilaun House B, Level 2, 111 St Stephen's Green, Dublin 2 D02 VN51, Ireland.

E-mail address: marybfitzsimons@rcsi.ie (M. Fitzsimons).
While integrated PCC is a key feature of current healthcare reform policies, this does not mean it will automatically translate into healthcare practice [16]. Coordinating care around the needs of the individual patient demands integrating knowledge and practice through collaboration both within and across professional, disciplinary, organizational, and sectoral boundaries $[2,6,17,18]$. It also requires HCPs to embrace new ways of working to drive better value within the healthcare system. However, a variety of coexisting realities for the actors involved may present multiple barriers to the necessary collaborative practice and desired innovation [2,19]. Exploring these social factors is fundamental to understanding the challenge of moving from statements of intent within policy to meaningful implementation [16].

As part of the ongoing national healthcare reform, the Irish Health Service Executive (HSE) established the National Clinical Programmes (NCP) in 2010 to drive service improvements in specific clinical domains [20]. Epilepsy care was one of the areas targeted by the NCPs for reform [20]. Characterized by the occurrence of recurrent unprovoked seizures, epilepsy can have significant quality of life consequences. Those with difficult to control or drug-resistant epilepsy have ongoing chronic care needs with increased likelihood of comorbidities, 
cognitive effects, social stigmatization, decreased life expectancy, and increased mortality risk $[21,22]$. Accordingly, the National Clinical Programme for Epilepsy (NCPE) describes a model that aims to achieve holistic integrated PCC [23-25]. Although its goals are generally agreed and welcomed by stakeholders, the steps required to realize the NCPE ambition and the preparedness of those concerned to make the journey are not clear.

The study presented in this paper aimed at understanding the readiness of the Irish epilepsy care ecosystem to realize the benefits of an integrated PCC model as advocated in current healthcare policy. Using a qualitative ethnographic investigation, the study focuses on the perspective of the HCP to learn about prevailing behaviors, attitudes, and values. Ultimately, the goal was to use learning from this investigation to inform the pathway to integrated PCC implementation.

\section{Methods}

\subsection{Study design}

This study is part of a wider exploration of the epilepsy care ecology in Ireland which was conducted to understand both HCPs, and people with epilepsy's experiences of PCC and integrated care in practice. Through the lens of those involved in its provision, ethnographic fieldwork was used to explore the everyday practice and nature of transactions in epilepsy care. Direct observation by researchers within the physical space of healthcare delivery helped clarify the meaning and context of epilepsy care (Table 1). In parallel, semi-structured one-to-one interviews were conducted to learn about PCC barriers and facilitators through the lived experience of HCPs (Table 2). To capture the fullest range of data, a stratified sample of epilepsy services representing a range of HCPs (Table 2), a variety of clinic types (Table 1), and covering different geographical locations (Tables 1 and 2) were recruited to the study.

\subsection{Study setting, participants, and data collection}

\subsubsection{Observations}

Over $100 \mathrm{~h}$ of nonparticipatory, documented observations were conducted at four hospital-based epilepsy centers across three regions in Ireland (Table 1). Settings observed included the waiting room of epilepsy outpatient clinics, patient with epilepsy and family group education sessions provided by HCPs, rapid access seizure clinics, one-toone face-to-face consultations between clinicians and patients, and virtual clinics conducted by telephone known as the telephone advice line (TAL). Researchers engaged in both passive observations, where they positioned themselves unobtrusively in the study setting, and shadowed some clinicians, with their agreement, as they navigated the epilepsy care setting. Field notes on observations of behaviors and interactions, layout of clinic rooms, work spaces and waiting areas, and the emotional tone of the environment were documented. As it is considered a key enabler in healthcare reform, the degree to which
Table 2

Interview participants.

\begin{tabular}{lccc}
\hline Role & ID & Sex & Site (see also Table 1) \\
\hline Epileptologists & E1 & Male & Hospital Site A \\
& E2 & Male & Hospital Site C \\
Advanced Nurse & E3 & Male & Hospital Site D \\
Practitioners/ Registered & ANP1 & Female & Hospital Site B \\
Nurse & ANP2 & Female & Hospital Site B \\
& ANP3 & Female & Hospital Site D \\
& ANP4 & Female & Hospital Site D \\
General Practitioners & RN1 & Female & Hospital Site D \\
& GP1 & Male & Non-hospital based \\
& GP2 & Female & Primary Care \\
& GP3 & Female & \\
Healthcare Managers & GP4 & Male & \\
& HCM1 & Male & Non-hospital based HSE \\
& HCM2 & Female & senior management. \\
& HCM3 & Female & \\
\hline
\end{tabular}

technology tools, such as electronic patient records (EPRs), play a part in healthcare provision was also noted.

\subsubsection{Interviews}

Semi-structured interviews were also conducted with healthcare professionals and managers $(n=15)$ (Table 2 ) about their everyday experience of delivering services and care for people with epilepsy. The interviewees included general practitioners (GPs) representing primary care $(n=4)$, consultant epileptologists $(n=3)$, advanced nurse practitioners (ANPs)/registered nurses representing the hospitalbased specialist epilepsy care perspective $(n=5)$, and senior healthcare managers $(n=3)$ representing the HSE which provides all of Ireland's public health services in hospitals and communities across the country. Interviews were conducted at a location convenient to the interviewee, were audio recorded and lasted between 45 and $60 \mathrm{~min}$.

The interview questions were guided by the following topics which were based on key dimensions of PCC [11-13,26]:

- taking a holistic approach to healthcare needs;

- treating the patient as an expert;

- recognizing patient autonomy;

- experience of sharing responsibility and decision-making;

- maintaining dignity and respect for patients;

- use of information and communication technology to support care needs.

\subsubsection{Data analysis}

Recordings of interviews were transcribed verbatim, and each participant was given a unique identifier. All interview transcripts and observation field notes were deidentified, collated, and analyzed using NVivo. Through a multiphased thematic analysis [27], the everyday tensions between the aims of the NCPE model of care [23] and participants' realities of healthcare delivery were inductively examined. Data analysis involved the following stages: data familiarization, identification of a thematic framework, indexing and

Table 1

Observation log.

\begin{tabular}{|c|c|c|c|c|c|}
\hline Site & Clinician-patient consultation & Outpatient waiting room & Telephone advice line & Group education clinic & Rapid access seizure clinic \\
\hline $\begin{array}{c}\text { A } \\
\text { (Region } 1 \text { hospital) }\end{array}$ & $\mathrm{N}$ & Y & Y & $\mathrm{Y}$ & Y \\
\hline $\begin{array}{c}\text { B } \\
\text { (Region } 1 \text { hospital) }\end{array}$ & $\mathrm{Y}$ & Y & $\mathrm{Y}$ & $\mathrm{N}$ & Y \\
\hline $\begin{array}{c}\text { C } \\
\text { (Region } 2 \text { hospital) }\end{array}$ & Y & Y & $\mathrm{Y}$ & $\mathrm{N}$ & $\mathrm{N}$ \\
\hline $\begin{array}{c}\text { D } \\
\text { (Region } 3 \text { hospital) }\end{array}$ & $\mathrm{N}$ & Y & Y & $\mathrm{N}$ & $\mathrm{N}$ \\
\hline
\end{tabular}


coding, and reviewing and refining of themes. The latter stages involved repeated group sessions where the full team of researchers came together to discuss and compare analyses of ethnographic observations and interviews to reach consensus on final data interpretations. Analysis was ongoing from when the first set of data became available and continued over the complete course of data collection.

\subsubsection{Ethical considerations}

Ethical approval for the project was granted by the Research Ethics Committees of participating sites (universities and clinical sites).

\section{Findings}

The findings indicate that while there is a person-centered ambiance, there are nontrivial limits to the readiness of the epilepsy care environment to fully meet the integrated PCC aspirations of healthcare reform. Nevertheless, HCPs demonstrate a willingness to advance PCC. These limits and tensions therein are illustrated under the following four key themes that emerged from the data: (i) unconscious bias and underdeveloped healthcare partnerships;, (ii) moving toward coordinated care;, (iii) managing contradictory consequences of innovation;, and (iv) balancing efficiency and effectiveness.

\subsection{Unconscious bias and underdeveloped healthcare partnerships}

While the data illustrated biases held by participants that could hinder development of the interprofessional, interorganizational, or patient-clinician alliances required to accomplish integrated PCC, it also showed their recognition that they could do better. For example, a hospital-based consultant epileptologist expressed a reluctance to discharge patients back to primary care while at the same time considering the need to change their practice:

I don't (discharge) because GPs often won't take them... They are not comfortable... if you ask the average GP they will say that people with epilepsy should be under the care of a neurologist... the problem is just the number of people with epilepsy... So it is very hard to discharge patients... what I might say is, "Listen, if you are in trouble call the nurses"... Maybe that is something I need to change, more proactive discharging [E3].

Similarly, a view held by a GP that comprehensive care is their dominion may represent both a barrier to interorganizational collaboration and a simultaneous expression of willingness to ensure care is delivered in the most appropriate setting for the patient:

I think as a GP we don't get to treat single disease, we don't have single disease clinics... people come into us and you have no idea what is wrong with them... the first thing any trained GP will say, "And what else, and what else?"... I see general practice almost means the same as holistic care [GP1].

Depending on their perspective, HCPs' opinions about the behaviors and expectations of the patient may further thwart care integration. A participating ANP noted:

... (patients are) very reluctant (to be discharged from the hospitalbased specialist service to primary care) because they feel once they go, they won't get back in [ANP4].

However, by engaging the patient as a partner in meaningful decision-making, such opinions can be elucidated and may help to develop better patient confidence in the wider collaborative care network. For example, during a group education session for people with epilepsy and their families held at a hospital-based specialist center, researchers observed a consultant epileptologist acknowledging the importance of integrating the experiential knowledge of the patient into the care team:

I have no idea what it's like to live with epilepsy... You are the experts... I have never had epilepsy... I'm an expert in medicine... [E1].

\subsection{Moving toward coordinated care}

The findings illustrate that HCPs experience a tension between providing a timely response to patient needs and taking time to improve the interprofessional reciprocity that is bubbling under the surface. In recalling a telephone conversation with a GP who was seeking advice about a person with epilepsy, an ANP realized the danger of creating a dependency on the hospital-based service rather than enabling a better primary-specialist shared care approach:

The GP is the gatekeeper, he has the comorbidities... he has the whole history there... that I can't see, I am the wrong person to make a decision. That person is physically in front of him, he can examine them, take a history, he has a duty of care... All you are doing is taking ownership of their problems and you don't build up any confidence in that person to actually resolve their own problem. So what do you do? You create a dependency [ANP1].

Likewise, a limited understanding of, or communication with, services beyond their own frame of reference can result in hospital-based services addressing a patient need that may more appropriately be managed in the community, as illustrated in the quote below from an ANP:

... it can be an alarm, somebody wanted an alarm there recently to video their seizures and they wanted a letter from us. Where is the social worker for applying for aids and appliances in the social welfare department? Why is it a nursing job... we actually don't have the knowledge to do it...you can't forget who is outside the walls of a hospital and there are lots of people who know these people apart from us. We think we know them well... [ANP1].

A statement by a hospital-based consultant epileptologist also illustrates how one-way communication reinforces fragmented care:

$99 \%$ of the time that I see a patient, I write a letter to the GP. I receive no letters from the GPs about their interaction with patients except a referral letter [E1].

Recognizing how a lack of proper coordination can result in suboptimal patient care and needless waste of finite resources, an ANP also stated:

It is complex... healthcare isn't simple, there is a lot of people employed in it, there is a lot of roles and a lot of responsibility and a lot of overlap and a lot of duplication... [ANP1].

It's supposed to be shared care [ANP, Site A observation].

While the requirement for better coordination and partnerships in care is appreciated by HCPs, understanding how these might be shaped remains a question:

... there should be some element of boundaries and (recognition) that the patient and doctor have different roles [GP2].

I try to make the decisions... you have to be a little bit paternalistic... I try to involve patients in decision-making... there is always more you can do... I try to give care to a population of people and be 
available to them all the time but not necessarily go to the so-called ends of the earth for an individual person [E3].

... but a partnership doesn't mean handing and investing all the power in one of the partners, it is more about... acknowledging the expertise, the role that each has... [HCM1].

\subsection{Managing contradictory consequences of innovation}

Observational data demonstrated that the Irish epilepsy care domain has adopted new ways of working and technological innovations with the aim of creating better value for both healthcare recipients and health service funders. Such initiatives are promoted in health service reform policy including the NCPE model of care. The national epilepsy EPR [28] and nurse led TAL are two such examples witnessed by researchers.

The observational data revealed how the EPR enables better continuity and coordination of care, both key PCC features, by ensuring patient information is available when and where needed. However, as expressed by ANPs, the EPR can also impact on effective communication as it diverts focus and attention away from the patient to enable data input. As demonstrated in one example below, this prompted an ANP to periodically reassure the patient:

Don't mind me looking at the screen, I am listening [ANP, Site B observation].

Typically, HCPs acknowledged both advantages and disadvantages with the EPR:

The benefits of the EPR far outweigh its problems... I can get all the info I need before I ring (via telephone) someone back [ANP, Site B observation].

However, as stated by a healthcare manager, a lack of interoperability between health information systems, such as the epilepsy EPR, diminishes the possibility for service integration:

... we do have our challenges in terms of our siloed ways of working... within a care division... our own individual professions... across hospital and community, and also in terms of data. So, we have got really good (IT) systems in primary care, they don't talk to (IT) systems in hospital... [HCM2].

As illustrated in the observation data, this poor information technology (IT) interoperability can lead to frustration among clinicians regarding availability and access to information:

... five patients so far today without access to scans and reports... how can we... treat patients without all the information?... I'll have to write and get the scans, how many months will that take? [E, Site B observation].

Meanwhile, findings regarding the epilepsy TAL revealed tensions between supply and demand. The aim of the epilepsy TAL is to deliver virtual clinics, where appropriate, by moving away from traditional face-to-face patient-clinician encounters; reduce unnecessary hospital visits; improve access to care; and increase value for money. The nature of the TAL service and the fact that it can be accessed from any location means HCPs consider it to be very person-centered as evidenced in the following excerpts:

They (the patient) are in their home, they are not in a clinic room... they have my full attention on the phone and you get to talk to them for a little bit longer on the phone... I find sometimes they are a little bit more open... [ANP4]. (via telephone)... we get to know them (the patient) in a very short time... we build a picture of what their life is, what is impacting on their life... [ANP3].

Another ANP highlighted that patients can leave a message and expect a return call:

... The response time is almost instantaneous depending on the complexity of the (reason for the) call... There is two things... the complexity of the call and... the level of experience of the person who is using (operating the TAL) the phone [ANP2].

Healthcare providers place importance on the TAL as a timely access point to specialist services for people with epilepsy. Additionally, observational data revealed that during clinical encounters, the epileptologists frequently advised patients to "give us a call". However, while appreciated as value-adding, the TAL was also seen sometimes as intense and unpredictable. Observations of ANPs operating the TAL demonstrated a relatively unfavorable working environment. In one site, ANPs were observed working in a cramped and stuffy office, not insulated from the sounds of the hospital or the outside (screeching seagulls) with the sun intensely shining through the window.

No one likes working on the TAL... so tiring and so busy... Every time I look at the phone, the bloody light is on (indicates message waiting) [ANP, Site B observation].

Another ANP enters and works TAL, comments; "we pick up a lot of the pieces". [ANP, Site B observation]

Across all of the study sites, a demanding succession of TAL calls, often of a complex nature, was observed frequently preventing the ANPs from taking a proper break, or even drinking a cup of coffee while working. Calls could come from multiple stakeholders including patients, GPs, and pharmacists for example. One of the sites had recently changed from a live call system to the ANP responding to voicemails left by callers.

The message service works well but you are never finished with it... that red light just keeps blinking... (messages require logging before responding)... been here until 6 or 7 just logging calls, never mind returning them... no idea how many messages there are... That light flashing, it's just so stressful [ANP, Site A observation].

\subsection{Balancing efficiency and effectiveness}

Observations and participant interviews highlighted how a frantic pace or time pressure in the clinical setting can inhibit personcenteredness and create frustration for clinicians as they try to balance the organizational requirement for efficiency with the patient need for effectiveness. The challenges HCPs experience in this regard can impact on the patient, the HCP themselves and/or their family, and the healthcare system as demonstrated in the following extracts:

... you have to remember there is a whole load of people waiting in a waiting room, getting frustrated because you are spending longer with one person and they are being delayed etc. So, it is a tightrope and a balancing act [GP3].

I don't have the time to do the work we are already doing... I don't even have the time to think about it... I keep taking on more work...we are doing things here that may or may not be working. I have no way of auditing anything; no time to follow through; no time to reflect [ANP, Site A observation]. 
The environment is not conducive to the patient being relaxed, it's a lack of resources... pace is the priority... [ANP1].

I have learned the hard way... it is not right to just impose a certain ten minutes, that is not the way life is... sometimes if a person wants to talk, you have to give them that time... if a patient spends half an hour with me then they have an expectancy that that is how much time they can spend with me every time... they wish to see me every time they come because we have built up a rapport... And that is not possible... I don't think I manage my time particularly well [E2].

My clinic used to start at 13:30... and would sometimes go on until 19:00 or $20: 00 \ldots$ I was flexible to allow people to talk or to allow myself to address things... (but) it had a lot of negative effects... people who were seen at 18:00 or 19:00 were very unhappy to be seen at that time... the nurses who were in the outpatients at 18:00 or 19:00 were very unhappy about being here... And my own family was very unhappy about me returning home late [E3].

Clinics are rushed. If you are well, you get 10 minutes. The health system is under resourced, understaffed and overbooked... I'm trying to do three or four things... It's a pain trying to balance things [ANP, Site B observation].

The system puts us under so much pressure and stress... We have to see so many patients; there aren't enough doctors and nurses [E, Site B observation].

The preceding excerpts comprehensively emphasize that while HCPs want to be person-centered and accommodate individual patient needs, the pressure to meet demands on health services within a finite timeframe poses a challenge to meeting this goal.

\section{Discussion}

Healthcare is a hugely complex system made up of a number of moving, evolving, and interdependent elements [29]. Therefore, the challenge of translating reform policies to action, while simultaneously maintaining day-to-day healthcare and service delivery, is not insignificant [16]. In the context of healthcare reform in Ireland $[20,23,24,30,31]$ and from the perspective of the HCP, this ethnographic study examined the current Irish epilepsy care landscape, and the motivations, values, and expectations of the actors involved. The study was prompted by a desire to develop a realistic roadmap for implementing a new model of integrated PCC for people with epilepsy in Ireland [23].

While the findings of the study demonstrate examples of a person-centered approach to care, they highlight coexisting pressures against advancing and optimizing the integrated PCC aspirations of healthcare reform. Assumptions explicit and implicit in the NCPE model of care about the readiness of the clinical environment to adopt and execute its recommendations are not borne out by the evidence. Although the model advocates integration, boundaries perpetuated within and between professions, disciplines, organizations, and sectors are resulting in missed opportunities for collaborative practice and consequent limits to the optimal coordination of patient care. Technological and service innovation aimed at creating better healthcare partnerships is at times resulting in greater dependencies rather than the intended clinician-clinician shared care and clinician-patient shared decisionmaking paradigm. Demand for pace and productivity is experienced by HCPs as running counter to the principles of PCC.

Given theories of boundaries and the recognized "labour of division" [17] undertaken by professions to build its field of knowledge and expert authority, a tendency toward territorialism observed in this study is not surprising. Transforming the resulting entrenched fragmented practices requires active change management to create spaces for communication and to build the alliances required for integrated PCC [14,32]. Similarly, without meaningful shared governance across organizational and/or sectoral boundaries, opportunities for identifying common ground, setting shared priorities, and building trust and mutuality are thwarted [7].

Adoption of new ways of working and new technologies by HCPs to support integrated PCC, particularly in hospital settings was demonstrated in this study. The TAL is enabling delivery of care when and where needed while the EPR facilitates timely sharing and exchange of patient information to enable integration of epilepsy care. However, unintended consequences of these initiatives were also identified. The EPR related ergonomic and interoperability issues pose a challenge to HCPs capacity to fully realize integrated PCC benefits. A recent New Yorker article similarly reported dissatisfaction among clinicians who feel that instead of making care easier, digitization is trapping them behind their computer screens [33]. Likewise, its responsiveness together with poor workplace aesthetics can result in stressful workload for HCPs delivering the TAL service. Given the growing awareness of innovation-related clinician burnout and its association with poor patient satisfaction and quality of care [34,35], careful implementation of healthcare innovations is required to reduce or prevent such unplanned effects.

The effects of neoliberalism on healthcare, similar to all public service delivery, have demanded the measurement of efficiency, with outcomes based on quantifiable activities [36] such as the number of patients seen, or the number of procedures carried out by the HCP during a clinical session. As a consequence, HCP work can become relatively task-oriented to meet the desired productivity [36]. Although the leadership role of ANPs in driving collaborative epilepsy care in Ireland has been recognized [24], an everyday focus on the management or mismanagement of time poses a challenge to fully realizing the goal. In this study, and as also found in Gachoud et al. and Sharp et al. [3,36], HCPs indicated that the tension between the time required to identify and respond to the individual patient's needs and the pressure to meet workload targets presents a considerable barrier to delivering holistic integrated PCC.

\section{Limitations}

This ethnographic analysis of the epilepsy care ecosystem in Ireland focused not only on the perspective of the HCP predominantly from the setting of hospital-based specialist epilepsy centers, but also on GPs and senior healthcare managers. A number of HSE regions were represented in the data. To gain a more comprehensive view an understanding of further healthcare settings and the perspective of other stakeholders, must also be considered. This should include, the person with epilepsy, their family and/or care partners, allied health professionals, community resource officers, non-consultant hospital doctors, primary care nurses, healthcare administrators and managers at different levels. As part of the wider project from which this study emanated some of these are being explored, with companion manuscripts about the patient and their family/care partner perspective and intersectoral hospital-community collaboration in preparation.

It is noted that sampling of HCPs is limited to those willing to engage and, therefore, there is an element of self-selection. The presence of researchers may have encouraged a tendency to complain rather than discuss successes. However, interactions throughout the study were numerous and varied enough to see HCPs as committed professionals who deliver a high standard of care within the limits of a system that often frustrates them. In using an ethnographic approach, the study did not seek to generalize as it focuses in a specific area of chronic care in Ireland. Nevertheless, the findings should be transferrable to other similarly complex chronic disease domains that struggle with multidimensional reform challenges. 


\section{Conclusions}

In the journey from policy to the practice of integrated PCC, multiple tensions collide. Firstly, tension between the generalized patient and the individual patient within policy creates a challenge to those tasked with its implementation [26], chiefly, because policy aims to improve care and services for all patients while simultaneously personalizing care for individuals.

Secondly, the pressure on HCPs to preserve health service continuity while simultaneously affecting reform of its structures and processes to drive quality improvement creates further tensions. For example, breaking down siloed behaviors and practices; creating the necessary governance structures; providing appropriate change-management support and resources; creating the essential reflective time and space to engage staff in incremental and iterative improvement initiatives will not happen spontaneously. Given this range of tensions and apparent contradictions, comprehensively achieving policy goals may not be realistic. Lastly, policy should be recognized as documents of good intentions, as suggested by Holt et al. [16], which discursively contain the values, rather than necessarily the steps, to guide implementation and readiness for reform.

\section{Acknowledgments}

The authors thank the employees of the Health Service Executive and independent practitioners who participated in this ethnographic study.

\section{Funding}

This research was funded by the Health Research Board Ireland (Research Collaborative for Quality and Patient Safety Grant no. RCQPS-2015-1).

\section{Declaration of interest}

The authors report no conflicts of interest. The authors alone are responsible for the content and writing of the article.

\section{References}

[1] Angus L, Valentijn PP. From micro to macro: assessing implementation of integrated care in Australia. Aust J Prim Health 2018;24:59-65.

[2] Auschra C. Barriers to the integration of care in inter-organisational settings: a literature review. Int J Integr Care 2018;18:1-14.

[3] Gachoud D, Albert M, Kuper A, Stroud L, Reeves S. Meanings and perceptions of patient-centeredness in social work, nursing and medicine: a comparative study. J Interprof Care 2012;26:484-90.

[4] Gauld R. The theory and practice of integrative health care governance. J Integr Care 2017;25:61-72.

[5] Huzzard T, Hellström A, Lifvergren S. Whole system in the room: toward systems integration in healthcare. Health Commun 2018;33:800-8.

[6] Kodner DL, Spreeuwenberg C. Integrated care: meaning, logic, applications, and implications - a discussion paper. Int J Integr Care 2002;2:e12.

[7] Nicholson C, Hepworth J, Burridge L, Marley J, Jackson C. Translating the elements of health governance for integrated care from theory to practice: a case study approach. Int J Integr Care 2018;18:11.

[8] Strandberg-Larsen M, Krasnik A. Measurement of integrated healthcare delivery: a systematic review of methods and future research directions. Int J Integr Care 2009;9.
[9] Sturmberg JP, O'Halloran DM, Martin CM. Understanding health system reform complex adaptive systems perspective. J Eval Clin Pract 2012;18:202-8.

[10] Swanson RC, Cattaneo A, Bradley E, Chunharas S, Atun R, Abbas KM, et al. Rethinking health systems strengthening: key systems thinking tools and strategies for transformational change. Health Policy Plan 2012;27:iv54-61.

[11] World Health Organisation. Framework on integrated, people-centred health services. Report by the Secretariat. Geneva: WHO; 2016.

[12] Håkansson Eklund J, Holmström IK, Kumlin T, Kaminsky E, Skoglund K, Höglander J, et al. "Same same or different?" A review of reviews of person-centered and patientcentered care. Patient Educ Couns 2019 Jan;102(1):3-11.

[13] Naldemirci Ö, Lydahl D, Britten N, Elam M, Moore L, Wolf A. Tenacious assumptions of person-centred care? Exploring tensions and variations in practice. Health 2018; 22:54-71.

[14] Naldemirci Ö, Wolf A, Elam M, Lydahl D, Moore L, Britten N. Deliberate and emergent strategies for implementing person-centred care: a qualitative interview study with researchers, professionals and patients. BMC Health Serv Res 2017;17:527.

[15] Willis CD, Best A, Riley B, Herbert CP, Millar J, Howland D. Systems thinking for transformational change in health. Evid Policy 2014;10:113-26.

[16] Holt DH, Rod MH, Waldorff SB, Tjornhoj-Thomsen T. Elusive implementation: an ethnographic study of intersectoral policymaking for health. BMC Health Serv Res 2018;18:54.

[17] Liberati EG, Gorli M, Scaratti G. Invisible walls within multidisciplinary teams: disciplinary boundaries and their effects on integrated care. Soc Sci Med 2016; 150:31-9.

[18] Fitzsimons M, Normand C, Varley J, Delanty N. Evidence-based models of care for people with epilepsy. Epilepsy Behav 2012;23:1-6.

[19] Kaats E, Opheij W. Creating conditions for promising collaboration: alliances, networks, chains, strategic partnerships. Heidelberg New York: Springer; 2014.

[20] Darker CD, Nicolson GH, Carroll A, Barry JM. The barriers and facilitators to the implementation of National Clinical Programmes in Ireland: using the MRC framework for process evaluations. BMC Health Serv Res 2018;18:733.

[21] Fisher RS, Acevedo C, Arzimanoglou A, Bogacz A, Cross JH, Elger CE, et al ILAE official report: a practical clinical definition of epilepsy. Epilepsia 2014; 55:475-82.

[22] Laxer KD, Trinka E, Hirsch LJ, Cendes F, Langfitt J, Delanty N, et al. The consequences of refractory epilepsy and its treatment. Epilepsy Behav 2014;37:59-70.

[23] Health Service Executive. The National Clinical Programme for Epilepsy: model of care. National Clinical Programme for Epilepsy HSE; 2016.

[24] Higgins A, Downes C, Varley J, Doherty CP, Begley C, Elliott N. Rising to the challenge: epilepsy specialist nurses as leaders of service improvements and change (SENsE study). Seizure 2018;63:40-7.

[25] Higgins A, Downes C, Varley J, Tyrell A, Normand C, Doherty CP, et al. Patients with epilepsy care experiences: comparison between services with and without an epilepsy specialist nurse. Epilepsy Behav 2018;85:85-94.

[26] Mead N, Bower P. Patient-centredness: a conceptual framework and review of the empirical literature. Soc Sci Med 2000;51:1087-110.

[27] Braun V, Clarke V. Using thematic analysis in psychology. Qual Res Psychol 2006;3: 77-101.

[28] Fitzsimons M, Dunleavy B, O'Byrne P, Dunne M, Grimson J, Kalra D, et al. Assessing the quality of epilepsy care with an electronic patient record. Seizure 2013;22: 604-10.

[29] Greenhalgh T, Papoutsi C. Studying complexity in health services research: desperately seeking an overdue paradigm shift. BMC Med 2018;16:1-6.

[30] Burke S, Barry S, Siersbaek R, Johnston B, Ní Fhallúin M, Thomas S. Sláintecare - a ten-year plan to achieve universal healthcare in Ireland. Health Policy 2018 Dec; 122(12):1278-82.

[31] Ireland. Oireachtas Committee on the future of healthcare Sláintecare report. Dublin: Houses of the Oireachtas; 2017.

[32] Young J, Egan T, Jaye C, Williamson M, Askerud A, Radue P, et al. Shared care requires a shared vision: communities of clinical practice in a primary care setting. J Clin Nurs 2017;26:2689-702.

[33] Gawande A. Why doctors hate their computers. The New Yorker; 2018.

[34] Bodenheimer T, Sinsky C. From triple to quadruple aim: care of the patient requires care of the provider. Ann Fam Med 2014;12:573-6.

[35] Drummond D. Physician burnout: its origin, symptoms, and five main causes. Fam Pract Manag 2015;22:42-7.

[36] Sharp S, McAllister M, Broadbent M. The tension between person centred and task focused care in an acute surgical setting: a critical ethnography. Collegian 2018;25:11-7. 